\title{
Coexistence of metallic edge states and anti-ferromagnetic ordering in correlated topological insulators
}

\author{
A. Amaricci, ${ }^{1}$ A. Valli, ${ }^{1}$ G. Sangiovanni, ${ }^{2}$ B. Trauzettel,${ }^{2}$ and M. Capone ${ }^{1}$ \\ ${ }^{1}$ Scuola Internazionale Superiore di Studi Avanzati (SISSA), and Consiglio Nazionale delle Ricerche, \\ Istituto Officina dei Materiali (IOM), Via Bonomea 265, 34136 Trieste, Italy \\ ${ }^{2}$ Institut für Theoretische Physik und Astrophysik, \\ Universität Würzburg, Am Hubland, D-97074 Würzburg, Germany
}

(Dated: May 11, 2018)

\begin{abstract}
We investigate the emergence of anti-ferromagnetic ordering and its effect on the helical edge states in a quantum spin Hall insulator, in the presence of strong Coulomb interaction. Using dynamical mean-field theory, we show that the breakdown of lattice translational symmetry favours the formation of magnetic ordering with non-trivial spatial modulation. The onset of a non-uniform magnetization enables the coexistence of spin-ordered and topologically non-trivial states. An unambiguous signature of the persistence of the topological bulk property is the survival of bona fide edge states. We show that the penetration of the magnetic order is accompanied by the progressive reconstruction of gapless states in sub-peripherals layers, redefining the actual topological boundary within the system.
\end{abstract}

\section{INTRODUCTION}

Understanding the impact of strong electron-electron interactions on Topological Insulators (TIs) is an open challenge $^{1-13}$ which holds the potential for the discovery of novel states of matter. One of the most subtle issues is that, while intrinsic electron-electron correlation effects may have a non-trivial interplay with topological properties, strong interactions typically favour different kinds of spontaneous symmetry breaking and the formation of long-range-ordered states (e.g. spin, charge or orbital ordering).

The paradigmatic case for non-frustrated correlated insulators is the anti-ferromagnetic (AFM) ground-state resulting from a local Hubbard repulsion. If we consider TIs, we can expect the magnetic ordering to obliterate the non-trivial topological character because of the breaking of the time-reversal symmetry (TRS). ${ }^{14-16}$ Suitable topological properties can however be preserved if an alternative or a residual symmetry persists. For commensurate AFM states a symmetry playing the role of timereversal can be realized by combining complex conjugation and lattice translations. ${ }^{17,18}$ Such symmetry gives rise to a $\mathbf{Z}_{2}$-classification in three dimensions but not in two.

The interplay between topological properties and correlation-driven AFM acquires further depth in a finite system, whose boundaries can host peculiar edge states, and at the same time they show stronger electron correlation effects ${ }^{19-25}$. In this article, we explore this situation by studying AFM ordering in the BernevigHughes-Zhang (BHZ) model, ${ }^{16}$ supplemented by a local interaction $^{6,10,25}$ on a stripe with open boundaries along one spartial direction using Dynamical Mean-Field Theory (DMFT). ${ }^{26,27}$

For a strong enough interaction the system undergoes a first-order transition to an AFM state. Near the transition the magnetization along the confined direction de- viates from a simple staggered pattern, allowing for a coexistence with a persisting topological state. Surprisingly, the helical edge states ${ }^{28-30}$ survive in the presence of AFM ordering for a large window of the interaction strength. When the external layers become insulating the edge states penetrate the "sub-peripheral" layers, redefining the actual boundaries of the TI. This edge reconstruction is similar to that taking place near a Mott insulator $^{23-25}$ which, however, does not break any of the symmetries protecting the topological state.

The novel boundary layers not only separates the topological bulk from the trivial ordered region, but also marks a dramatic change in the many-body character of the system. While the insulating AFM layers can be basically described in terms of Hartree-Fock theory, ${ }^{31}$ the inescapable presence of the gapless edge states turns out to bring correlation effects, beyond Hartree-Fock picture, back into the game. The outcome of correlations, topology and boundary effects is therefore a strongly correlated AFM state which remains metallic with gapless edge states.

The topological nature of the system arises from the residual $U(1)$ symmetry, corresponding to the spinrotation around the $z$-axis, as the magnetic ordering does not have in-plane components. In general, the $U(1)$ invariant topological states are referred to as spin-Chern insulators (SCIs). ${ }^{32-34}$ The emergence of an interaction driven SCI in the bulk have been addressed before using different approaches ${ }^{1,32,35}$. However, the analysis of the corresponding edge states in a finite system is still lacking. Our calculations demonstrate that a SCI is realized in a finite geometry near a correlation driven AFM transition.

The article is organized as follows. In Sec. II, we introduce the interacting BHZ model (that allows for magnetic ordering) and discuss the method of solution. In Sec. III, we present the main results of our work. In particular, we investigate the antiferromagnetic ordering in a stripe geometry with distinction of bulk and edge properties. 


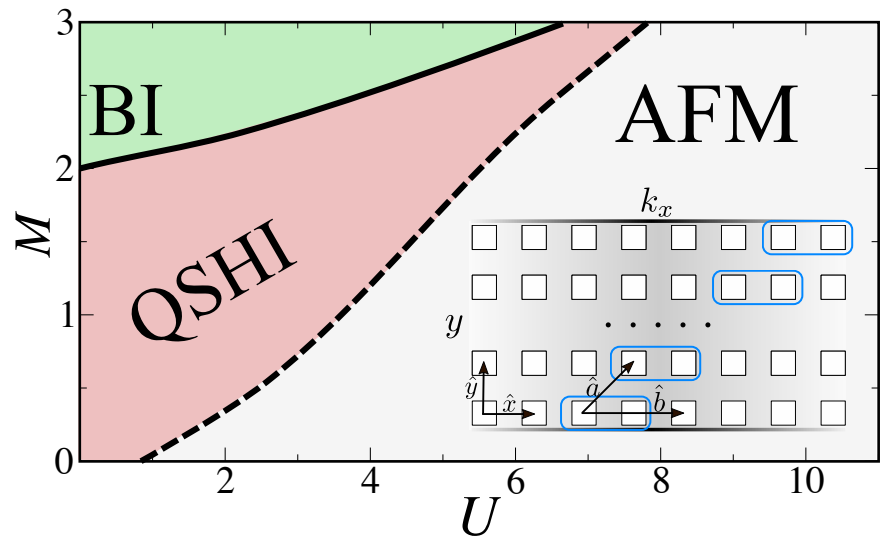

FIG. 1. (Color online) Main panel: $M-U$ phase diagram of the model with periodic boundary conditions in both directions. For small $U$, the system is either a trivial band insulator (BI) or a quantum spin Hall insulator (QSHI). For larger interaction strength, an AFM solution becomes stable. The ordered phase is separated from the QSHI by a first-order transition. Inset: schematic structure of the main model in a stripe geometry with a unit cell hosting two inequivalent sites.

Finally, in Sec. IV, we conclude and point out interesting perspectives.

\section{INTERACTING BHZ MODEL}

We consider a two-orbital BHZ model in two dimensions in presence of a local interaction term. ${ }^{6,10,25}$ The Hamiltonian can be written in terms of the identity $\Gamma_{0}=\mathbb{1} \otimes \mathbb{1}$ and the following $4 \times 4$ matrices: $\Gamma_{x}=\sigma_{z} \otimes \tau_{x}$, $\Gamma_{y}=-\mathbb{1} \otimes \tau_{y}, \Gamma_{5}=\mathbb{1} \otimes \tau_{z}$ and $\Gamma_{\sigma}=\sigma_{z} \otimes \mathbb{1}$. Here, $\sigma_{x, y, z}$ and $\tau_{x, y, z}$ are two sets of Pauli matrices acting, respectively, on the spin and orbital sector.

If periodic boundary conditions (PBC) are assumed in both spatial directions, the non-interacting part of the Hamiltonian can be diagonalized in momentum space: $H=\sum_{\mathbf{k}} \psi_{\mathbf{k}}^{\dagger} H_{\mathbf{k}} \psi_{\mathbf{k}}$, where the spinor $\psi_{\mathbf{k}=\left(k_{x}, k_{y}\right)}$ collects the operators $c_{\mathbf{k} \alpha \sigma}$ destroying electrons at the orbital $\alpha=$ 1,2 with spin $\sigma=\uparrow, \downarrow$, while $H_{\mathbf{k}}=E(\mathbf{k}) \Gamma_{5}+\lambda \sin \left(k_{x}\right) \Gamma_{x}+$ $\lambda \sin \left(k_{y}\right) \Gamma_{y}$ and $E(\mathbf{k})=M-\epsilon\left[\cos \left(k_{x}\right)+\cos \left(k_{y}\right)\right]$. This Hamiltonian is invariant under TRS and conserves $S_{z}$, i.e. it has $U(1)$ spin rotation symmetry.

In order to describe the effects of AFM ordering, we consider a unit cell with two inequivalent sites, $\Lambda=A, B$ (see inset in Fig.1). The unit cell tiles the lattice along the two vectors $\hat{a}=a_{\text {lat }}(1,1), \hat{b}=a_{\text {lat }}(2,0)$, with $a_{\text {lat }}$ is the lattice constant which we assume as our unit length.

In this paper we solve the model in a geometry where we have open boundaries and a finite number $L_{y}$ of layers along the $\hat{a}$ direction, while PBC are assumed along the $\hat{b}$ direction ("stripe geometry") as shown in the inset of Fig.1. In the hybrid basis $\left(k_{x}, y\right)$, the model Hamiltonian
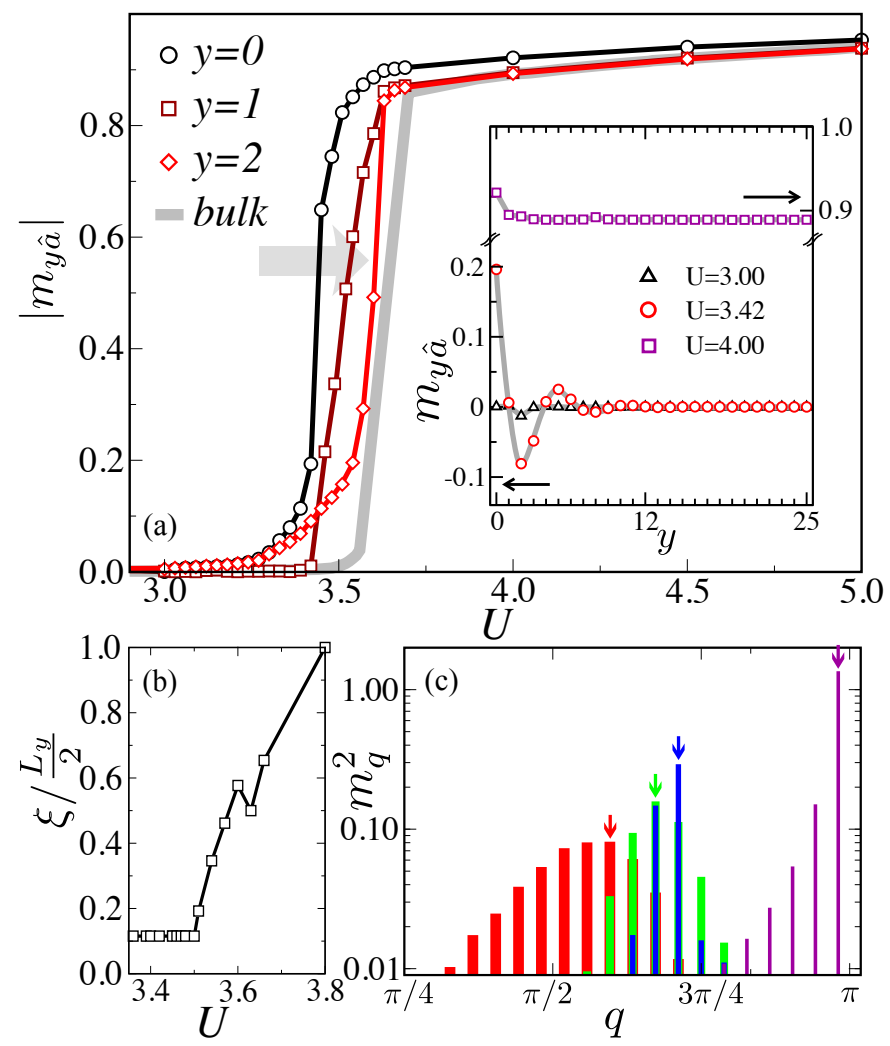

FIG. 2. (Color online) (a): Magnetization $\left|m_{y \hat{a}}\right|$ along the $\hat{a}$ direction as a function of $U$ and for the first three layers $y=0,1,2$. Data are shown for the choice of site $\Lambda=A$. The thick (gray) line corresponds to the magnetization behavior in the homogeneous case. Inset: Layer resolved magnetization $m_{y}$ for $\Lambda=A$ and three values of the interaction strength $U$. (b): Correlation length of the magnetization profile $\xi$ as a function of $U$. Data are in units of the half size of the stripe $L_{y}$ along the transverse direction. (c): Evolution of the Fourier spectrum of the magnetization $m_{q}^{2}$ across the AFM transition.

takes the form

$$
\begin{aligned}
H= & \sum_{k_{x}, y, y^{\prime}} \Psi_{k_{x} y}^{\dagger} \mathbf{M}\left(k_{x}\right) \delta_{y y^{\prime}} \Psi_{k_{x} y^{\prime}}+ \\
& \sum_{k_{x} y, y^{\prime}}\left(\Psi_{k_{x} y}^{\dagger} \mathbf{A}\left(k_{x}\right) \delta_{y+1 y^{\prime}} \Psi_{k_{x} y^{\prime}}+\text { H.c. }\right)+H_{\mathrm{int}},
\end{aligned}
$$

where $y=0, \ldots, L_{y}-1$ is the coordinate in $\hat{y}$ direction, i.e. the layer index, $\Psi_{k_{x} y}=\left(\psi_{\mathbf{k}, A}, \psi_{\mathbf{k}, B}\right)$, and

$$
\begin{aligned}
\mathbf{M}\left(k_{x}\right) & =\left[\begin{array}{cc}
\hat{m} & \hat{t}_{x}+\hat{t}_{x}^{\dagger} e^{i 2 k_{x}} \\
\hat{t}_{x}^{\dagger}+\hat{t}_{x} e^{-i 2 k_{x}} & \hat{m}
\end{array}\right], \\
\mathbf{A}\left(k_{x}\right) & =\left[\begin{array}{cc}
\hat{0} & \hat{t}_{y}^{\dagger} e^{i k_{x}} \\
\hat{t}_{y}^{\dagger} e^{-i k_{x}} & \hat{0}
\end{array}\right]
\end{aligned}
$$

with $\hat{m}=M \Gamma_{5}, \hat{t}_{x}=-\frac{\epsilon}{2} \Gamma_{5}+i \frac{\lambda}{2} \Gamma_{x}, \hat{t}_{y}=-\frac{\epsilon}{2} \Gamma_{5}+i \frac{\lambda}{2} \Gamma_{y}$. In the remainder of this article, we set $\epsilon$ as the energy unit.

In Eq. (1), $H_{\text {int }}$ describes a local Coulomb interaction with both inter- and intra-orbital repulsion and the 
Hund's coupling $J$, taking into account the exchange effect which favors high-spin configurations. In terms of local operators, $\hat{N}=\sum_{\mathbf{i j}} \Psi_{\mathbf{i}}^{\dagger} \Gamma_{0} \delta_{\mathbf{i j}} \Psi_{\mathbf{j}}, \hat{S}_{z}=\frac{1}{2} \sum_{\mathbf{i}} \Psi_{\mathbf{i}}^{\dagger} \Gamma_{\sigma} \delta_{\mathbf{i j}} \Psi_{\mathbf{j}}$, $\hat{T}_{z}=\frac{1}{2} \sum_{\mathbf{i}} \Psi_{\mathbf{i}}^{\dagger} \Gamma_{5} \delta_{\mathbf{i j}} \Psi_{\mathbf{j}}$, the interaction term reads

$$
H_{\mathrm{int}}=(U-J) \frac{\hat{N}(\hat{N}-1)}{2}-J\left(\frac{\hat{N}^{2}}{4}+\hat{S}_{z}^{2}-2 \hat{T}_{z}^{2}\right),
$$

where $U$ is the strength of the electron-electron interaction and $\Psi_{\mathbf{i}=x, y}=\sqrt{\frac{2 \pi}{V}} \sum_{k_{x}} e^{-i k_{x} \cdot x} \Psi_{k_{x} y}{ }^{36}$. In the following, we fix $\lambda=0.3$ and a relatively large ratio $J=U / 4$ but none of our results are specific to this choice.

We solve the interacting problem non-perturbatively using DMFT ${ }^{10,11,25,37,38}$ at zero temperature. In order to capture the different behavior between bulk and boundaries, we rely on its real-space extensions for inhomogeneous systems. ${ }^{39-45}$ In this framework, the interaction effect is contained in a diagonal but layer-dependent selfenergy function $\boldsymbol{\Sigma}_{y}(\omega)$, bearing the correct spin-orbital structure.

\section{AFM ORDERING AND METALLIC EDGE STATES}

The presence of strong electronic interaction usually favours the emergence of an instability towards longrange ordered states at low temperature. For half-filled bipartite lattices the leading instability is towards a staggered AFM ordering. Our system makes no exception to this generic consideration. We clarify this point in Fig.1, reporting the phase diagram of the model in an infinite system with $\mathrm{PBC}$ in both directions. ${ }^{10}$ In the non-interacting regime $(U=0)$ the system undergoes a topological quantum phase transition at $M=2$ from a band-insulator (BI) for $M>2$ to a quantum spin Hall insulator (QSHI) at $M<2$. These two phases are adiabatically continued into separate regions of the diagram for finite values of the interaction. The QSHI region is topologically non-trivial hence the $\mathbf{Z}_{2}$ invariant is $\nu=\left(\mathcal{C}_{\uparrow}-\mathcal{C}_{\downarrow}\right) / 2=1$, where $\mathcal{C}_{\sigma}$ is the Chern number for a given spin orientation. ${ }^{7}$ In both the QSHI and the BI regions the ground state is paramagnetic and TRS is preserved. However, for larger values of the interaction strength the system undergoes a transition from the paramagnetic QSHI to an AFM phase. The magnetic transition takes place at $U=U_{c}^{\text {homo }}$ and it is of the first order (see Fig.2(a)). In the homogeneous case the AFM phase is topologically trivial as TRS is explicitly broken. Notably, we cannot exclude the possible existence of a SCI phase in the coexisting region near the critical point, as pointed out in Ref. 46. The very existence of such non-trivial metastable phase requires a fine-tuning of the model parameters. We will show below that an extended SCI phase, characterizing by a finite decaying magnetization in the bulk, naturally emerges in a finite geometry as a result of strong correlations.
We now consider the system on the stripe geometry introduced above. To characterize the magnetic nature of the solution we consider the local magnetization $m_{\mathbf{i}}=$ $\frac{1}{2} \sum_{\alpha}\left(n_{\mathbf{i} \alpha \uparrow}-n_{\mathbf{i} \alpha \downarrow}\right)$, where $n_{\mathbf{i} \alpha \sigma}$ is the local occupation at the site $\mathbf{i}$, for the orbital $\alpha$ with spin $\sigma$. In a finite system, the lattice translational symmetry is broken, leading to a substantial reduction of the kinetic energy near the boundaries. This makes the sites near the edge effectively more correlated. ${ }^{20,21,25,43}$ Hence, we expect the external layers to build up a magnetic order more rapidly with respect to the bulk as the interaction increases. ${ }^{44}$

This effect is illustrated in Fig.2(a) here we plot the layer-resolved absolute value of the magnetization $\left|m_{y \hat{a}}\right|$, along the diagonal direction $\hat{a}$ for the sublattice $\Lambda=A$. This quantity would be a constant as a function of $y$ in an ideal Néel antiferromagnet. Here we plot the absolute value to focus on the way the interaction leads to the formation of magnetic moments in the first layers. As a function of $U$, the initially non-magnetic system at weak coupling is progressively driven into an ordered state by increasing the interaction strength. However, the most external layer, $y=0$, reaches the saturated value for the magnetization for smaller interactions than the more internal layers. The behavior of $m_{y \hat{a}}$ shows a sequential behavior with respect to the different layers, near the saturation point. In this regime, few external magnetic layers coexist with a paramagnetic "bulk". Further increasing the interaction strength, the AFM ordering penetrates the inner layers. For interactions larger than a critical value $U>U_{c}^{\text {stripe }} \simeq U_{c}^{\text {homo }}$ the whole system becomes AFM.

Interestingly, the spatial distribution of the magnetization has a non-trivial behavior which we explore In the inset of Fig.2(a). Here we report the evolution of the magnetization $m_{y \hat{a}}$ as a function of the layer index $y$ along the $\hat{a}$-direction and for the sublattice $\Lambda=A$. Along the periodic direction a perfect Néel ordering is imposed.

For weak interactions, the magnetization is negligible everywhere reflecting the non-magnetic nature of the solution in this regime. By increasing the correlation strength in the regime $U<U_{C}^{\text {stripe }}$, the system develops a finite magnetization near the boundaries which is exponentially suppressed in the bulk on a length scale $\xi$. Interestingly, the magnetization shows a non-trivial spatial modulation before the staggered pattern (which corresponds to a constant magnetization with our notation) sets in. In this regime, the ordered boundary layers coexist with a yet non-magnetic bulk. Only for a larger interaction strength, the system eventually develops a nearly constant and flat magnetization profile $m_{y \hat{a}}$, corresponding to the complete transition of the stripe to an AFM state.

In order to get insight into the development of the AFM state and the non-trivial spatial distribution of the order parameter, we investigate both the evolution of the correlation length in transverse direction $\xi$ and the Fourier decomposition of the magnetization profile, i.e. $m_{q}=\sqrt{\frac{2 \pi}{L_{y}}} \sum_{y} e^{i q y} m_{y}$ for $q=\frac{n \pi}{L_{y}}$ and $n=0, \ldots, L_{y}-1$. 


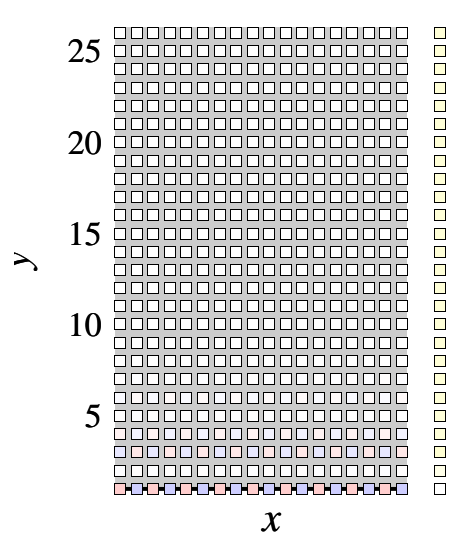

(a)

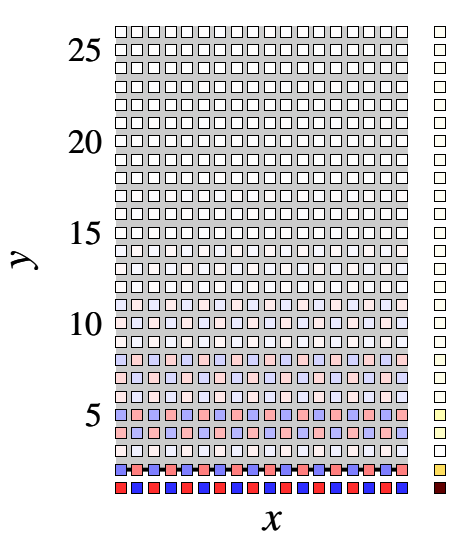

(b)

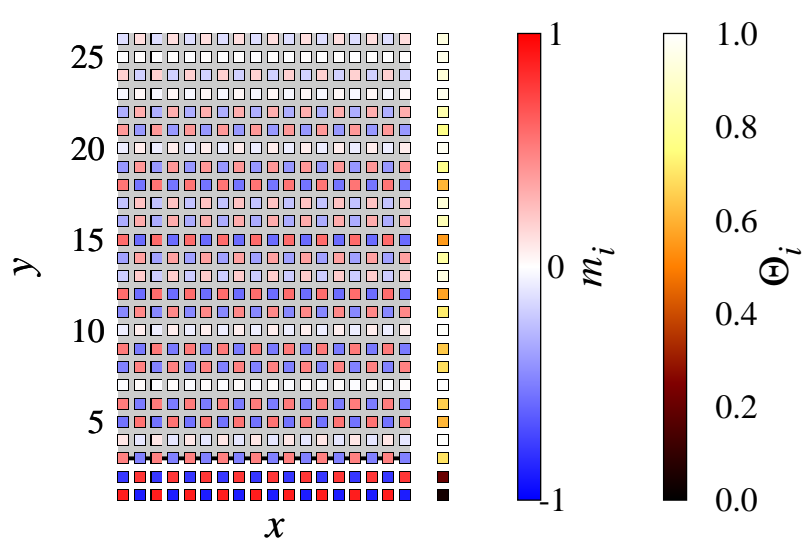

(c)

FIG. 3. (Color online) Spatially resolved local magnetization $m_{i}$ and correlation strength $\Theta_{i}$ in the stripe for increasing values of the interaction strength across the AFM transition, $U=3.42$ (a), 3.51 (b) and 3.60 (c). The figures correspond to half of the stripe. Data are displayed for $L_{y}=52$. The color scale for the magnetization and the correlation strength are reported on the vertical bars on the right. The (black) solid lines indicate the location of the reconstructed edge states.

The results for these two quantities are reported in Fig.2(b)-(c). The following scenario emerges. Approaching the AFM transition, the correlation length increases from few $(\xi \simeq 2)$ to many lattice units. Thus, enhancing the interaction strength, an increasing number of layers from the boundary to the bulk order magnetically. When the correlation length reaches the half-size of the stripe, $\xi \simeq L_{y} / 2$, the two AFM fronts "propagating" from the opposite boundaries overlap. Beyond this point the system becomes entirely AFM. Simultaneously, the spectral distribution of the order parameter undergoes a dramatic change. Initially, the distribution has a wide "bell"-like shape, spread over many momenta and centered near $q_{\text {peak }} \simeq \frac{\pi}{2}$. Increasing $U$ the width of the distribution reduces while the peak contribution increases its weight. Approaching the transition the spectral distribution of the AFM order parameter becomes entirely dominated by the peak contribution, which in turn approaches the value $q_{\text {peak }}=\pi$. Eventually, at $U>U_{c}^{\text {stripe }}$ the magnetization converges towards the form $m_{\mathbf{i}}=e^{i \mathbf{Q i}} M$, where $\mathbf{i}=(x, y)$ and $\mathbf{Q}=(\pi, \pi)$ is the ordering vector of the Néel AFM state, as expected in the homogeneous limit, i.e. $\mathrm{PBC}$ in all directions.

The construction of the AFM state in the stripe comes with the progressive breakdown of TRS. In the absence of such symmetry the conventional QSHI is modified and its helical states at the boundary change nature. However, we found that near the AFM transition the system remains in a topological state, endowed with vestigial edge states. Such gapless modes are protected by the residual $U(1)$ symmetry associated with the spin-rotation around the easy axis. If this symmetry was broken, a gap would open for the edge states. The presence of the $U(1)$ symmetry is enough to stabilize the topological state against the magnetic ordering, leading to a progressive reconstruction of the edge states, ${ }^{25}$ and the consequent contraction of the topological bulk, as the AFM order pen- etrates into the system. In order to illustrate this effect we report in Fig.3(a)-(c) the evolution of the spatial distribution of the magnetization and of the reconstructed edge states. For small values of the interaction strength the staggered magnetization is weak and localized at the boundary layer, where it coexists with the gapless mode. As the interaction is increased the AFM order slowly builds up and progressively penetrates into the stripe. As the magnetization reaches its maximum value at the most external layer, a gap in the energy spectrum opens and the gapless edge states shifts inward. In this regime of the interaction strength $U<U_{c}^{\text {stripe }}$, the system hosts a non-trivial topological bulk with a residual weak magnetization distribution, separated from the trivial AFM part at the most external layers by a conductive state located in "sub-peripherals" layers.

The discontinuity in the topological character at the edge layer is accompanied by a dramatic change in the nature of the ground state. The topologically non-trivial bulk shows a strongly correlated nature. We estimate the correlation strength in terms of the electronic self-energy as:

$$
\Theta_{\mathbf{i}}=\frac{\operatorname{Tr}\left[\Gamma_{5} \operatorname{Re} \boldsymbol{\Sigma}_{\mathbf{i}}(0)-\Gamma_{5} \boldsymbol{\Sigma}_{\mathbf{i}}^{\mathrm{HF}}\right]}{\operatorname{Tr}\left[\Gamma_{5} \operatorname{Re} \boldsymbol{\Sigma}_{\mathbf{i}}(0)\right]}
$$

where $\boldsymbol{\Sigma}_{\mathbf{i}}^{\mathrm{HF}}=\lim _{n \rightarrow \infty} \operatorname{Re} \boldsymbol{\Sigma}_{\mathbf{i}}\left(i \omega_{n}\right)$. Deep inside the AFM ordered state, the DMFT yields very similar results to the static Hartree-Fock theory, and the ground state has a low degree of electronic correlation, i.e. the electronic states are very close to single-particle states ${ }^{31}$ Near the transition, the penetration of the AFM ordering in the strongly interacting topological bulk gives rise to a state that is simultaneously correlated and magnetic. As such, the nature of this state goes beyond a conventional Hartree-Fock description. In order to illustrate this point we show in Fig. 3 the behavior of $\Theta_{\mathbf{i}}$ on one half of the stripe along the transverse direction. At $U<U_{c}^{\text {stripe }}$ the 


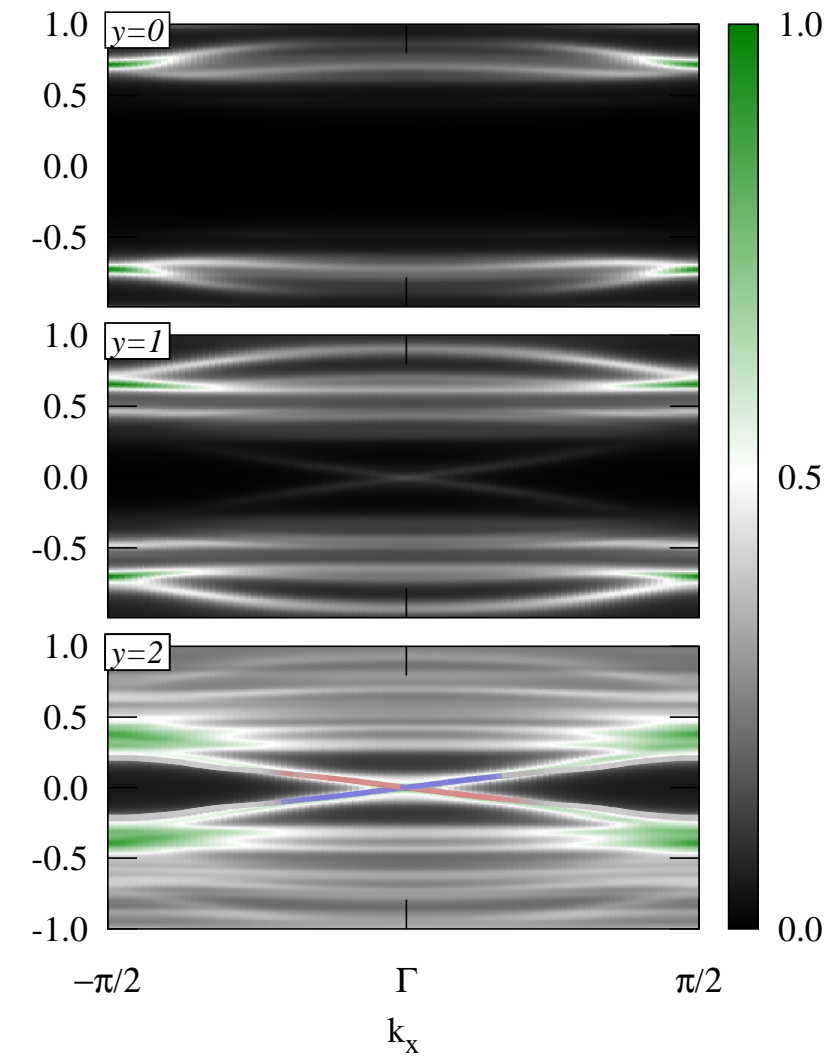

FIG. 4. (Color online) Layer- and $k_{x}$-resolved spectral functions $A_{y}\left(k_{x}, \omega\right)$ for $y=0$ (top), $y=1$ (center) and $y=2$ (bottom). Data are for $U=3.60$. The solid lines indicate the dispersion of the gapless edge states located at the third layer.

correlation strength is nearly at its maximum at all layers. Approaching the magnetic transition, $\Theta_{\mathbf{i}}$ is strongly suppressed in the trivial AFM boundaries, and it remains finite at the reconstructed edges as well as in the topological bulk, despite the progressive penetration of magnetization. Remarkably, we observe the same spatial modulation between the magnetization and the suppression of $\Theta_{\mathbf{i}}$. The system retains a substantial degree of correlation until the AFM correlation length reaches the value $\xi \sim L_{y} / 2$, and the whole system becomes a trivial AFM insulator.

Finally, we can characterize the edge reconstruction phenomena studying the spatial evolution of the $k_{x^{-}}$and layer-resolved spectral function: $A_{y}\left(k_{x}, \omega\right)=$ $-\frac{1}{4 \pi} \sum_{\alpha, \sigma} \operatorname{Im} G_{\alpha, y}\left(k_{x}, \omega+i 0^{+}\right)$. In Fig.4, we report the low-energy part of $A_{y}\left(k_{x}, \omega\right)$ around the $\Gamma$ point for the first three layers at one edge of the stripe, near the AFM transition. For $U=3.60<U_{c}^{\text {stripe }}$, the gapless helical states at the $y=0$ layer have collapsed leaving behind a spectral gap associated to the AFM ordering. While some sub-gap spectral weight is developed, due to hybridization with the bulk layers, in the second interior layer $(y=1)$, it is only at the third layer $(y=2)$ that we can find a new pair of gapless states, renormalized by electronic correlations separating the trivial AFM insulator from the topologically non-trivial bulk. The situation is identical at the opposite side of the stripe.

\section{CONCLUSIONS AND OUTLOOK}

We have investigated the effect of AFM ordering caused by strong local interactions on the topological insulating state of the BHZ model and in particular on the gapless edge states.

Solving the model on a stripe with open boundaries, we have pointed out the existence of a first-order transition to a Néel type AFM state. The transition is preceded by a regime in which the magnetic order has a non-trivial spatial modulation. Unlike in conventional insulators, the onset of inhomogeneous magnetic order does not destroy entirely the topological insulating state which, instead, survives in the bulk. The opening of a magnetic gap in the most external layer is accompanied by the creation of novel helical edge states in the immediately adjacent layers, which become the actual topological boundary of the system. These edge states are protected by a residual $U(1)$ symmetry. Thus, our findings unveil a rich phenomenology of the edge states of interacting spin-Chern insulators. In particular our calculations suggest a novel way to induce a metallic state in an antiferromagnet caused by strong electronic correlations.

A conventional way to drive an AFM into a metallic state is through hole doping. This comes with a number of fascinating consequences, such as spin-charge separation in one dimension and confined spin polarons. It is even believed to cause high-temperature superconductivity in two dimensions. Yet, injecting holes in an AFM state inevitably spoils the long-range order and eventually destroys it. In our work, we find that symmetry protection of the edges in a topologically non-trivial AFM driven by strong correlation enables for the spatial coexistence of metallic regions with long-range ordered magnetization. We suggest that this effect should also be realized at the interface between a trivial AFM insulator and a TI. The proximity of the two phases could allow for an atomically thin AFM metallic state. This heterostructure could be of relevance for the emerging research field of AFM spintronics ${ }^{47,48}$.

\section{ACKNOWLEDGEMENTS}

We thank J.C. Budich for useful discussions and suggestions as well as the critical reading of the manuscript. We thank M.Vojta for interesting discussions. A.A. thanks M.Fabrizio for helpful discussions. A.A. and M.C. acknowledge support from the Seventh Framework Programme FP7, under Grant No. 280555 "GO FAST", and the H2020 Framework Programme, under ERC Ad- 
vanced Grant No. 692670 "FIRSTORM". A.V. acknowledges financial support from the Austrian Science Fund (FWF) through the Erwin Schrödinger fellowship J3890N36. A.A., A.V. and M.C. also acknowledge financial support from MIUR PRIN 2015 (Prot. 2015C5SEJJ001) and SISSA/CNR project "Superconductivity, Ferroelectricity and Magnetism in bad metals" (Prot. 232/2015). G.S. and B.T. acknowledge financial support by the DFG (SPP 1666 on "Topological Insulators" and SFB 1170 "ToCoTronics").
1 Stephan Rachel and Karyn Le Hur, "Topological insulators and mott physics from the hubbard interaction," Phys. Rev. B 82, 075106 (2010).

2 M. Hohenadler, T. C. Lang, and F. F. Assaad, "Correlation effects in quantum spin-hall insulators: A quantum monte carlo study," Phys. Rev. Lett. 106, 100403 (2011).

3 M. Hohenadler and F. F. Assaad, "Luttinger liquid physics and spin-flip scattering on helical edges," Phys. Rev. B 85, 081106 (2012).

4 Y. Tada, R. Peters, M. Oshikawa, A. Koga, N. Kawakami, and S. Fujimoto, "Correlation effects in two-dimensional topological insulators," Phys. Rev. B 85, 165138 (2012).

5 Jan Carl Budich, Ronny Thomale, Gang Li, Manuel Laubach, and Shou-Cheng Zhang, "Fluctuation-induced topological quantum phase transitions in quantum spinHall and anomalous-Hall insulators," Phys. Rev. B 86, 201407 (2012).

6 Jan Carl Budich, Björn Trauzettel, and Giorgio Sangiovanni, "Fluctuation-driven topological Hund insulators," Phys. Rev. B 87, 235104 (2013).

7 M Hohenadler and F F Assaad, "Correlation effects in twodimensional topological insulators," Journal of Physics: Condensed Matter 25, 143201 (2013).

8 Hsiang-Hsuan Hung, Victor Chua, Lei Wang, and Gregory A. Fiete, "Interaction effects on topological phase transitions via numerically exact quantum Monte Carlo calculations," Phys. Rev. B 89, 235104 (2014).

9 Feng Lu, JianZhou Zhao, Hongming Weng, Zhong Fang, and Xi Dai, "Correlated Topological Insulators with Mixed Valence," Phys. Rev. Lett. 110, 096401 (2013).

10 A. Amaricci, J. C. Budich, M. Capone, B. Trauzettel, and G. Sangiovanni, "First-Order Character and Observable Signatures of Topological Quantum Phase Transitions," Phys. Rev. Lett. 114, 185701 (2015).

11 A. Amaricci, J. C. Budich, M. Capone, B. Trauzettel, and G. Sangiovanni, "Strong correlation effects on topological quantum phase transitions in three dimensions," Phys. Rev. B 93, 235112 (2016).

12 Bitan Roy, Pallab Goswami, and Jay D. Sau, "Continuous and discontinuous topological quantum phase transitions," Phys. Rev. B 94, 041101 (2016).

13 Pramod Kumar, Thomas Mertz, and Walter Hofstetter, "Interaction-induced topological and magnetic phases in the hofstadter-hubbard model," Phys. Rev. B 94, 115161 (2016).

14 C. L. Kane and E. J. Mele, " $Z_{2}$ Topological Order and the Quantum Spin Hall Effect," Phys. Rev. Lett. 95, 146802 (2005).

15 C. L. Kane and E. J. Mele, "Quantum Spin Hall Effect in Graphene," Phys. Rev. Lett. 95, 226801 (2005).

16 B. Andrei Bernevig, Taylor L. Hughes, and Shou-Cheng Zhang, "Quantum Spin Hall Effect and Topological Phase Transition in HgTe Quantum Wells," Science 314, 17571761 (2006).
17 Roger S. K. Mong, Andrew M. Essin, and Joel E. Moore, "Antiferromagnetic topological insulators," Phys. Rev. B 81, 245209 (2010).

18 Chen Fang, Matthew J. Gilbert, and B. Andrei Bernevig, "Topological insulators with commensurate antiferromagnetism," Phys. Rev. B 88, 085406 (2013).

19 Atsuo Shitade, Hosho Katsura, Jan Kuneš, Xiao-Liang Qi, Shou-Cheng Zhang, and Naoto Nagaosa, "Quantum spin hall effect in a transition metal oxide na iro $_{3}, "$ Phys. Rev. Lett. 102, 256403 (2009).

20 Giovanni Borghi, Michele Fabrizio, and Erio Tosatti, "Surface dead layer for quasiparticles near a mott transition," Phys. Rev. Lett. 102, 066806 (2009).

21 G. Borghi, M. Fabrizio, and E. Tosatti, "Strongly correlated metal interfaces in the Gutzwiller approximation. ," Phys. Rev. B 81, 115134 (2010).

22 G. Mazza, A. Amaricci, M. Capone, and M. Fabrizio, "Electronic transport and dynamics in correlated heterostructures," Phys. Rev. B 91, 195124 (2015).

23 Amal Medhi, Vijay B. Shenoy, and H. R. Krishnamurthy, "Synchronous and asynchronous mott transitions in topological insulator ribbons," Phys. Rev. B 85, 235449 (2012).

${ }^{24}$ H. Ishida and A. Liebsch, "Buried topological edge state associated with interface between topological band insulator and mott insulator," Phys. Rev. B 90, 205134 (2014).

25 A. Amaricci, L. Privitera, F. Petocchi, M. Capone, G. Sangiovanni, and B. Trauzettel, "Edge state reconstruction from strong correlations in quantum spin hall insulators," Phys. Rev. B 95, 205120 (2017).

26 Antoine Georges, Gabriel Kotliar, Werner Krauth, and Marcelo J. Rozenberg, "Dynamical mean-field theory of strongly correlated fermion systems and the limit of infinite dimensions," Rev. Mod. Phys. 68, 13-125 (1996).

27 G. Kotliar, S. Y. Savrasov, K. Haule, and et al., "Electronic structure calculations with dynamical mean-field theory," Rev. Mod. Phys. 78, 865 (2006).

28 Markus Knig, Steffen Wiedmann, Christoph Brne, and et al., "Quantum Spin Hall Insulator State in HgTe Quantum Wells," Science 318, 766-770 (2007).

29 A. Roth, C. Brüne, H. Buhmann, L. W. Molenkamp, J. Maciejko, X.-L. Qi, and S.-C. Zhang, "Nonlocal transport in the quantum spin hall state," Science 325, 294-297 (2009).

30 Ivan Knez, Rui-Rui Du, and Gerard Sullivan, "Evidence for helical edge modes in inverted InAs/GaSb quantum wells," Phys. Rev. Lett. 107, 136603 (2011).

31 G. Sangiovanni, A. Toschi, E. Koch, K. Held, M. Capone, C. Castellani, O. Gunnarsson, S.-K. Mo, J. W. Allen, H.-D. Kim, A. Sekiyama, A. Yamasaki, S. Suga, and P. Metcalf, "Static versus dynamical mean-field theory of mott antiferromagnets," Phys. Rev. B 73, 205121 (2006).

32 Tsuneya Yoshida, Robert Peters, Satoshi Fujimoto, and Norio Kawakami, "Topological antiferromagnetic phase in a correlated bernevig-hughes-zhang model," Phys. Rev. B 
87, 085134 (2013).

33 Yao-Hua Chen, Hsiang-Hsuan Hung, Guoxiong Su, Gregory A. Fiete, and C. S. Ting, "Cellular dynamical meanfield theory study of an interacting topological honeycomb lattice model at finite temperature," Phys. Rev. B 91, 045122 (2015).

34 Stephan Rachel, "Quantum phase transitions of topological insulators without gap closing," Journal of Physics: Condensed Matter 28, 405502 (2016).

35 S. Miyakoshi and Y. Ohta, "Antiferromagnetic topological insulator state in the correlated bernevig-hughes-zhang model," Phys. Rev. B 87, 195133 (2013).

36 This Hamiltonian only contains the "density-density" part of the Hund's exchange and neglects the so-called pairhopping and spin-flip terms. The robustness of the topological transitions in the BHZ model against the pair-hopping and spin-flip terms has been verified in Ref. 6 .

37 Massimo Capone, Luca de' Medici, and Antoine Georges, "Solving the dynamical mean-field theory at very low temperatures using the Lanczos exact diagonalization," Phys. Rev. B 76, 245116 (2007).

38 C. Weber, A. Amaricci, M. Capone, and P. B. Littlewood, "Augmented hybrid exact-diagonalization solver for dynamical mean field theory," Phys. Rev. B 86, 115136 (2012).

39 M. Potthoff and W. Nolting, "Metallic surface of a mott insulator ${ }^{\prime}$ mott insulating surface of a metal," Phys. Rev. B 60, 7834-7849 (1999).

40 J. K. Freericks, Transport in Multilayered Nanostructures (Imperial College Press, London, 2006).
41 M Snoek, I Titvinidze, C Töke, K Byczuk, and W Hofstetter, "Antiferromagnetic order of strongly interacting fermions in a trap: real-space dynamical mean-field analysis," New Journal of Physics 10, 093008 (2008).

42 A. Amaricci, A. Privitera, and M. Capone, "Inhomogeneous bcs-bec crossover for trapped cold atoms in optical lattices," Phys. Rev. A 89, 053604 (2014).

43 A. Valli, G. Sangiovanni, A. Toschi, and K. Held, "Correlation effects in transport properties of interacting nanostructures," Phys. Rev. B 86, 115418 (2012).

44 A. Valli, A. Amaricci, A. Toschi, T. Saha-Dasgupta, K. Held, and M. Capone, "Effective magnetic correlations in hole-doped graphene nanoflakes," Phys. Rev. B 94, 245146 (2016).

45 Angelo Valli, Adriano Amaricci, Valentina Brosco, and Massimo Capone, "Quantum interference assisted spin filtering in graphene nanoflakes," Nano Letters, Nano Letters 18, 2158-2164 (2018).

46 Tsuneya Yoshida, Robert Peters, Satoshi Fujimoto, and Norio Kawakami, "Topological antiferromagnetic phase in a correlated Bernevig-Hughes-Zhang model," Phys. Rev. B 87, 085134 (2013).

47 A. H. MacDonald and M. Tsoi, "Antiferromagnetic metal spintronics," Philosophical Transactions of the Royal Society of London A: Mathematical, Physical and Engineering Sciences 369, 3098-3114 (2011).

48 T. Jungwirth, J. Sinova, A. Manchon, X. Marti, J. Wunderlich, and C. Felser, "The multiple directions of antiferromagnetic spintronics," Nature Physics 14, 200-203 (2018). 